\title{
Animal Models of Neurological Disorders
}

\author{
Marie-Francoise Chesselet • S. Thomas Carmichael
}

Published online: 30 March 2012

(C) The American Society for Experimental NeuroTherapeutics, Inc. 2012

Animal modeling of human disease is a cornerstone to basic scientific studies of disease mechanisms and pre-clinical studies of potential therapies. Rapid progress in animal modeling has led to advancements in the understanding of fundamental disease mechanisms of many central nervous system (CNS) disorders, including initial cell death and later repair in stroke $[1,2]$, motor and nonmotor pathologies in Parkinson's Disease $[3,4]$, and axonal regeneration in peripheral and optic nerve injury [5, 6], among many others. Ideally, animal modeling produces basic insights, new views of the human disease, and preclinical trials of novel therapies. Much progress has been accomplished in that direction since the earlier coverage of this topic in the issue of Neurotherapentics in July 2005. Yet controversy and challenges in animal modeling of human CNS diseases continue to occur.

Negative views of animal modeling of neurological diseases often stem from failures in the application of an animal model to pre-clinical testing of candidate treatment molecules. In the stroke field, roughly 500 neuroprotective therapies that were seen as successful in rodent models of stroke subsequently failed at some stage of translation to humans, with only 1 treatment emerging as a new, approved therapy [7, 8]. In amyotrophic lateral sclerosis the mouse superoxide dismutase $1\left(\mathrm{SOD} 1^{\mathrm{G} 39 \mathrm{a}}\right)$ disease model has been the platform for the

M.-F. Chesselet $(\bowtie)$

Department of Neurobiology, Reed Neurological Research Center, B114, 710 Westwood Plaza,

Los Angeles, CA 90095, USA

e-mail: MChesselet@mednet.ucla.edu

S. T. Carmichael $(\bowtie)$

Department of Neurology,

David Geffen School of Medicine at UCLA,

710 Westwood Plaza,

Los Angeles, CA 90095, USA

e-mail: scarmichael@mednet.ucla.edu testing of hundreds of candidate therapies, with only 1 therapy (riluzole) making it into clinical practice with a modest functional benefit [9]. Indeed, a meta-analysis of treatment data with this mouse model led to predictions for success with a candidate therapy (minocycline [10]), which actually made patients worse in a subsequent clinical trial [11]. Importantly, many of these failures in animal models may relate to experimental design and data analysis rather than to the models themselves $[9,12]$. However, the use of models with uncertain mechanistic relevance to the human disease may also lead to disappointing results in clinical trials as illustrated by the reliance on toxin-based models for modeling Parkinson's disease and the failure of drugs that show neuroprotection in these models to provide benefits to patients [13]. It is clear that a careful study of animal modeling is necessary as these models become incorporated into the translational process of neurotherapeutic development and preclinical drug testing.

The review articles in this special issue of Neurotherapeutics focus on novel aspects of animal modeling for both basic scientific studies of disease and pre-clinical drug development. Authors were asked to focus on a critical assessment of a select number of new models rather than to provide comprehensive coverage of the field. Starting these reviews, Auvin et al. [14] discuss rules in animal modeling of pediatric epilepsy syndromes. This is a challenging field for animal modeling, because it needs to include not just the model of a progressive pathology, but also needs to take account of early brain development in the disease and differences in brain developmental timelines between the rodent and humans. Auvin et al. [14] elaborate on a set of 5 principles for pediatric epilepsy models, but ones that also apply more generally to an animal model of CNS disease. Höke [15] amplifies these animal modeling principles in a careful review of rodent models of peripheral neuropathy, with a focus on diabetic, chemotherapeutic, and human 
immunodeficiency virus peripheral neuropathies. Some of the most promising outcome measures for basic scientific discovery of molecular mechanisms and pre-clinical drug testing have emerged from studies in which human and rodent peripheral nerve innervation patterns can be directly evaluated in parallel outcome measures.

In single gene disease states, mouse transgenic modeling would seem to offer an ideal platform for exact molecular mimicry of the causal condition. In Huntington's disease, the genetic etiology of a cytosine-adenine-guanine (CAG) repeat expansion in the huntingtin gene has been known for some time [16]. However, models that broadly express the mutation have provided useful tools for understanding pathophysiology and testing drugs (more sophisticated genetic approaches that can be used to ask mechanistic questions about the role of specific cell populations in the disease process). Ehrlich [17] review data from different animal models and derived cell culture studies, and how these can inform cell autonomous and noncell autonomous interactions in the development of Huntington's disease pathology. In many cases, animal models of single gene disorders have not been simple to generate. In Machado-Joseph disease (spinocerebellar ataxia 3) variations in triplet nucleotide expansion in the ataxin 3 gene along with 2 isoforms of the gene product (mjd1a and ataxin3) lead to a complex clinical presentation, including variations in cerebellar, basal ganglia, corticospinal tract, oculomotor, and muscle signs [18]. Colomer Gould [19] describes distinct transgenic strategies that vary the gene promoter, triplet repeats, isoform, and conditional expression to model high intermediate and low severity phenotypes in this disease. On the other hand, rare genetic mutations have provided an unexpected window into the understanding of the largely nongenetic illness, Parkinson's disease. As reviewed by Chesselet et al. [4] strong evidence implicates alpha-synuclein, a rarely mutated protein, in the sporadic forms of Parkinson's disease. Accordingly, some genetic mouse models based on alpha-synuclein over-expression reproduce a wide array of Parkinson's disease-like deficits, opening the door to a better understanding of pathophysiology and providing a tool for preclinical drug testing in a relevant model of sporadic Parkinson's disease.

Single gene alterations in transgenic mouse models, however, rarely model the full extent or presentation of the disease in humans. Fremont and Khodakhah [20] describe the difficulties in modeling single gene disorders in focal dystonia in mice. However, a unique approach has produced a valid mouse model of dystonia by infusing an antagonist to the specific gene system in a genetically modified mouse: the sodium/potassium adenosine triphosphate-ase (ATPase) inhibitor oubain infused into cerebellum or basal ganglia of the Dyt12, sodium potassium ATPase mutated mouse. This model uses pharmacogenetics and site specific targeting to recapitulate most aspects of a human disease with multiple etiologies. From the other end of the human disease spectrum, an acute brain injury clearly has no genetic predilection. Shoch et al. illustrate the power of serial molecular analysis with mouse transgenics applied to a pathologically complex event, such as traumatic brain injury, that produces mechanistic results in studies of cell death and neuroplasticity. Finally, in complex human behavioral traits, such as addiction or affective behaviors, gene by gene studies in mouse models are severely limiting. Bubier and Chesler [21] describe integrative genetics and genomics that use careful genetic mapping of inbred strain hybrids to determine gene regions and gene expression patterns that underlie these complex behavioral phenotypes. This approach combines newly realized mouse genetics through bioinformatics processing to develop insights into the molecular underpinnings of behavioral traits and psychiatric disease.

The issue of brain size and complexity in modeling human disease is a recurring topic, loosely related as big brains versus small brains in animal models [22]. Neuroanatomical differences between humans and rodents make modeling some CNS diseases difficult. A principle example is in white matter structure and the organization of cortical projection systems. The amount of cerebral white matter in humans is roughly $4 \times$ $10^{6} \mathrm{~mm}^{3}$, compared with $10 \mathrm{~mm}^{3}$ in the mouse [23]. Certain diseases are particularly focused in white matter, such as subcortical stroke in the elderly, which leads to vascular dementia, and the white matter injury in the perinatal period, which leads to cerebral palsy. There is a lack of fundamental molecular data on these diseases because of previously poor animal modeling. With precise targeting, mouse white matter can serve as a locus for a focal stroke model that in many ways mimics human subcortical white matter stroke. Sozmen et al. describe a mouse subcortical white matter stroke, and review the field of white matter stroke models in rodents. A salient point from this review is that white matter stroke sets cellular events in motion that are clearly distinct from the more commonly modeled "grey matter" or cortical and striatal rodent strokes. However, there are such profound differences in white matter structure and brain development between the rodent and the human that specific elements of white matter disease require large animal models. Back et al. [24] describe the instrumented fetal sheep model of white matter injury and its ability to provide a platform for imaging, pathophysiology, and therapeutic trials as a relevant model for human perinatal white matter injury. This sheep model captures not only a complex and large subcortical white matter structure, but also a time course for white matter development that much more closely follows the human progression than does that of the rodent.

Large animal models of human CNS disease are most closely approximated in the nonhuman primate. The experimental infrastructure necessary to support such studies and the 
experimental models themselves require substantial resources and dedicated time and personnel. Yet the benefits in modeling biodistribution and mimicking the anatomical complexity of the human brain and spinal cord may justify such expense. Cook and Tymianski [25] review new primate models of stroke with a particular focus on the challenges in setting up such a model, and the benefits when imaging, tissue analysis, and long-term behavioral readouts can be synchronized in 1 single stroke model. The spinal cord injury field has seen the development of many different mouse and rat animal models. Several features of the human spinal cord are not seen in rodents. The corticospinal tract of course runs in a distinctly different site within the spinal cord of the rodent compared with humans, and the degree of motor neuron innervation, direct muscular versus intrinsic spinal network control, and postlesion plasticity differ between rodents versus primates [22, 26]. Importantly, the primate spinal cord contains extensive bilateral axonal collaterals compared with the rodent [27], which are likely to contribute to both normal and postinjury control of limb function, which is not present in rodents [26]. Nout et al. [28] describe a novel model of cervical spinal cord injury and forelimb control in the nonhuman primate that provides a basis for studies of regeneration and functional recovery of arm and hand control.

The issue of brain size and complexity in modeling human disease also extends down phylogenetic orders of magnitude: little brains versus connected groups of neurons. Although lacking the complex central interactions of an actual brain, lower vertebrate animal models of disease provide tractable testing of neuronal, glial, and vascular interactions within a connected and functional network. The worm has provided crucial insights into normal and pathological aging, rapid molecular characterization of candidate gene systems, and registered behavioral readouts. Dexter et al describe Caenorhabditis elegans modeling of movement disorders and the further use of the nematode for high throughput drug screening in a process that positions the worm model in a level above that of cell culture: an in vivo system for drug discovery. The studies in this review illustrate how the fast-moving gain and loss of function studies in multiple molecular pathways of a candidate gene system serially and in parallel can determine causal interaction in disease pathology.

Collectively, the reviews gathered in this special issue illustrate the ingenuity of investigators in addressing the momentous challenges of modeling human disorders in animals in a meaningful manner. It is easy to criticize individual models for their failure to reproduce all facets of the human disease, but this is to be expected. The more important question is whether the models are useful to better understand the mechanisms leading to the manifestations of neurological diseases, validate drug targets, and provide the necessary confidence that a compound or therapeutic approach will ultimately benefit patients. Critical evaluation of models remains paramount, but progress is being made daily as illustrated by these reviews.

Required Author Forms Disclosure forms provided by the authors are available with the online version of this article.

\section{References}

1. Carmichael ST. Animal models of stroke: size, mechanism and purpose. NeuroRx 2005;2:396-409.

2. Murphy TH, Corbett D. Plasticity during stroke recovery: from synapse to behaviour. Nat Rev Neurosci 2009;10:861-872.

3. Dunnett SB, Lelos M. Behavioral analysis of motor and non-motor symptoms in rodent models of Parkinson's disease. Prog Brain Res 2010;184:35-51.

4. Chesselet M-F, Richter F, Zhu C, Magen I, Watson MB, Subramaniam SR. A Progressive Mouse Model of Parkinson's Disease: The Thy1-aSyn Mice. Neurotherapeutics 2012. doi:10.1007/ s13311-012-0104-2 (in this issue).

5. Limb GA, Martin KR. Sixth ARVO/Pfizer Ophthalmics Research Institute Conference Working Group. Current prospects in optic nerve protection and regeneration: sixth ARVO/Pfizer Ophthalmics Research Institute conference. Invest Ophthalmol Vis Sci $2011 ; ; 52: 5941-5954$.

6. Wood MD, Kemp SW, Weber C, Borschel GH, Gordon T. Outcome measures of peripheral nerve regeneration. Ann Anat 2011;193:321333

7. O'Collins VE, Macleod MR, Donnan GA, Horky LL, van der Worp BH, Howells DW. 1,026 experimental treatments in acute stroke. Ann Neurol 2006;59:467-477.

8. Sena ES, van der Worp HB, Bath PM, Howells DW, Macleod MR. Publication bias in reports of animal stroke studies leads to major overstatement of efficacy. PLoS Biol 2010;8:e1000344.

9. Scott S, Kranz JE, Cole J, et al. Design, power, and interpretation of studies in the standard murine model of ALS. Amyotroph Lateral Scler 2008;9:4-15.

10. Benatar M. Lost in translation: treatment trials in the SOD1 mouse and in human ALS. Neurobiol Dis 2007;26:1-13.

11. Gordon PH, Moore DH, Miller RG, et al. Efficacy of minocycline in patients with amyotrophic lateral sclerosis: a phase III randomised trial. Lancet Neurol 2007;6:1045-1053.

12. Macleod MR, van der Worp HB, Sena ES, Howells DW, Dirnagl U, Donnan GA. Evidence for the efficacy of NXY-059 in experimental focal cerebral ischaemia is confounded by study quality. Stroke 2008;39:2824-2829.

13. Lohle M, Reichmann H. Clinical neuroprotection in Parkinson's disease - still waiting for the breakthrough. J Neurol Sci 2010;289:104-114.

14. Auvin S, Pineda E, Shin D, Gressens P, Mazarati A. Novel Animal Models of Pediatric Epilepsy. Neurotherapeutics 2012. doi:10.1007/s13311-012-0119-8 (in this issue).

15. Höke A. Animal Models of Peripheral Neuropathies. Neurotherapeutics 2012. doi:10.1007/s13311-012-0116-y (in this issue).

16. The Huntington's Disease Collaborative Research Group. A novel gene containing a trinucleotide repeat that is expanded and unstable on Huntington's disease chromosomes. Cell 1993;72:971-983.

17. Ehrlich ME. Huntington's Disease and the Striatal Medium Spiny Neuron: Cell-Autonomous and Non-Cell-Autonomous Mechanisms of Disease. Neurotherapeutics 2012. doi:10.1007/s13311012-0112-2 (in this issue).

18. Dürr A, Stevanin G, Cancel G, Duyckaerts C, Abbas N, Didierjean O, Chneiweiss H, Benomar A, Lyon-Caen O, Julien J, Serdaru M, Penet C, Agid Y, Brice A. Spinocerebellar ataxia 3 and Machado- 
Joseph disease: clinical, molecular, and neuropathological features. Ann Neurol 1996;39:490-499.

19. Colomer Gould VF. Mouse Models of Spinocerebellar Ataxia Type 3 (Machado-Joseph Disease). Neurotherapeutics 2012. doi:10.1007/s13311-012-0117-x (in this issue).

20. Fremont R, Khodakhah K. Alternative Approaches to Modeling Hereditary Dystonias. Neurotherapeutics 2012. doi:10.1007/ s13311-012-0113-1 (in this issue).

21. Bubier JA, Chesler EJ. Accelerating Discovery for Complex Neurological and Behavioral Disorders through Systems Genetics and Integrative Genomics in the Laboratory Mouse. Neurotherapeutics 2012. doi:10.1007/s13311-012-0111-3 (in this issue).

22. Courtine G, Bunge MB, Fawcett JW, et al. Can experiments in nonhuman primates expedite the translation of treatments for spinal cord injury in humans? Nat Med 2007;13:561566.

23. Zhang K, Sejnowski TJ. A universal scaling law between gray matter and white matter of cerebral cortex. Proc Natl Acad Sci U S A 2000;97:5621-5626.
24. Back SA, Riddle A, Dean J, Hohimer AR. The Instrumented Fetal Sheep as a Model of Cerebral White Matter Injury in the Premature Infant. Neurotherapeutics 2012. doi:10.1007/s13311-012-0108-y (in this issue).

25. Cook DJ, Tymianski M. Nonhuman Primate Models of Stroke for Translational Neuroprotection Research. Neurotherapeutics 2012. doi:10.1007/s13311-012-0115-z (in this issue).

26. Rosenzweig ES, Courtine G, Jindrich DL, et al. Extensive spontaneous plasticity of corticospinal projections after primate spinal cord injury. Nat Neurosci 2010;13:1505-1510.

27. Rosenzweig ES, Brock JH, Culbertson MD, et al. Extensive spinal decussation and bilateral termination of cervical corticospinal projections in rhesus monkeys. J Comp Neurol 2009;513:151-63.

28. Nout YS, Rosenzweig ES, Brock JH, Strand SC, Moseanko R, Hawbecker S, Zdunowski S, Nielson JL, Roy RR, Courtine G, Ferguson AR, Edgerton VR, Beattie MS, Bresnahan JC, Tuszynski MH. Animal Models of Neurologic Disorders: A NonhumanPrimate Model of Spinal Cord Injury. Neurotherapeutics 2012. doi:10.1007/s13311-012-0114-0 (in this issue). 